\section{Genome Update: Chromosome Atlases}

\section{Genomes of the month}

Six new bacterial genomes have been published since last month's Genome Update. The list is shown in Table 1 and includes a species that can grow happily in a refrigerator (the $\delta$-proteobacterium Desulfotalea psychrophila, with an optimal growth temperature of $10^{\circ} \mathrm{C}$, although it can grow at temperatures slightly below freezing!) and the actinobacterium Propionibacterium acnes, which can cause acne in humans. Let us hope that these two never cross, because the thought of a fridge with complexion problems is not very pleasant! The other genomes include that of the actinobacterium Leifsonia xyli, those of the firmicutes Mycoplasma mobile and Streptococcus pyogenes and that of an $\alpha$-proteobacterium, Rickettsia typhi. A brief overview of each of these genomes is given below.

D. psychrophila strain LSv $54^{\mathrm{T}}$ was isolated from permanently cold coastal sediments off the coast of Svaldbard, Norway.

$D$. psychrophila is the type species of a genus of sulfate-reducing bacteria, Desulfotalea. Cells of strain LSv $54^{\mathrm{T}}$ are thin, long and rod-shaped with an optimal doubling time of $27 \mathrm{~h}$ at $10^{\circ} \mathrm{C}$. This is the first bacterium to be sequenced that grows happily at such low temperatures. The genome consists of a main chromosome of 3.5 Mbp, a large plasmid of $121 \mathrm{kbp}$ and a smaller plasmid of about $15 \mathrm{kbp}$ (Rabus et al., 2004). About $27 \%$ of the proteins have no homologues in public databases. There are some differences in amino acid composition, such as a preference for cysteines, but any conclusions based on essentially one genome are difficult to draw (one 'datum point' compared to more than 100 other genomes); at any rate, it is not easy to tell whether this bias might also be explained by the difference in proteins used for sulfur metabolism. The genomes of more psychrophilic organisms need to be sequenced, as cold environments represent a large part of the Earth's biosphere, especially when one takes into account the oceans.

The plant pathogen L. xyli, although not a psychrophile, has an optimal growth temperature of only about $20^{\circ} \mathrm{C}$, and has the largest number of pseudogenes for any bacterial plant pathogen sequenced to date (307 of 2326 predicted ORFs) (Monteiro-Vitorello et al., 2004). L. xyli also has the lowest coding density (70.09\%) of the 196 prokaryotic genomes currently available to the public. Most bacterial genomes (167 of 177) have coding densities of at least $80 \%$ (see supplemental materials for a table of genomes sorted by coding density). Surprisingly few genes associated with pathogenicity were found in this genome ( 85 of the 2030 predicted genes). It seems that L. xyli can synthesize the plant hormone abscisic acid, which is a growth inhibitor; this could explain how this bacterium can cause stunted growth in plants.

The genome of M. mobile strain $163 \mathrm{~K}^{\mathrm{T}}$ is about $780 \mathrm{kbp}$ long and encodes 633 genes (Jaffe et al., 2004). It is very AT-rich (75\%), making it the third most AT-rich bacterial genome sequenced to date. M. mobile has only 28 tRNAs, the fewest number for any bacterium sequenced. More importantly, there is another 'first' for this genome, in that the authors also describe a detailed analysis of the proteome. In fact, proteomics data were used to help in annotation, and 26 genes that were not annotated originally were added. An amazing $88 \%$ of the predicted genes have been detected experimentally as proteins (Jaffe et al., 2004). The M. mobile genome lacks all the genes for the citric acid cycle and for de novo nucleotide and amino acid synthesis. One interesting aspect of this genome is a set of five genes called ' $r p$ ' (for long terminal repeat), which were found to be nearly perfect repeats, although the proteins have slightly different amino acid sequences and are expressed differently.

The genome of $P$. acnes strain KPA171202 is $2.6 \mathrm{Mbp}$ long and is predicted to contain 2333 genes (Brüggemann et al., 2004).

Increased cellular and humoral immunity to $P$. acnes has been observed in patients with severe acne, and the genome of this bacterium contains many potential cell-surface proteins with antigenic potential, including 25 genes with a C-terminal LPXTG type cell-wall sorting signal required for attachment of surface proteins. Some of these genes contain hypervariable stretches of Gs or Cs that can cause phase variation in the protein sequence.

R. typhi is an obligate intracellular parasite that causes murine typhus. The genome of $R$. typhi strain Wilmington ${ }^{\mathrm{T}}$ is $1 \cdot 1 \mathrm{Mbp}$ long, with an AT content of $71 \%$ (McLeod

\footnotetext{
Microbiology Comment provides a platform for readers of Microbiology to communicate their personal observations and opinions in a more informal way than through the submission of papers.

Most of us feel, from time to time, that other authors have not acknowledged the work of our own or other groups or have omitted to interpret important aspects of their own data. Perhaps we have observations that, although not sufficient to merit a full paper, add a further dimension to one published by others, or we may have a useful piece of methodology that we would like to share.

Guidelines on how to submit a Microbiology Comment article can be found in the Instructions for Authors at http://mic.sgmjournals.org

It should be noted that the Editors of Microbiology do not necessarily agree with the views expressed in Microbiology Comment.

Chris Thomas, Editor-in-Chief
} 
Table 1. Summary of the published genomes discussed in this Update

Note that the accession number for each chromosome is the same for GenBank, EMBL and the DDBJ.

\begin{tabular}{|lcccccc|}
\hline Name & Length & AT content (\%) & No. of genes & tRNAs & rRNAs & Accession no. \\
\hline Desulfotalea psychrophila LSv54 ${ }^{\mathrm{T}}$ & 3523383 & $53 \cdot 2$ & 3118 & 64 & 7 & CR522870 \\
Leifsonia xyli CTCB07 & 2584158 & $32 \cdot 3$ & 2030 & 45 & 1 & AE016822 \\
Mycoplasma mobile 163K & 777079 & $75 \cdot 1$ & 633 & 28 & 1 & AE017308 \\
Propionibacterium acnes KPA171202 & 2560265 & $40 \cdot 0$ & 2297 & 45 & 3 & AE017283 \\
Rickettsia typhi Wilmington ${ }^{\mathrm{T}}$ & 1111496 & $71 \cdot 1$ & 838 & 33 & 1 & AE017197 \\
Streptococcus pyogenes MGAS10394 & 1899877 & $61 \cdot 3$ & 1886 & 67 & 6 & CP000003 \\
\hline
\end{tabular}

et al., 2004). A three-way comparison of R. typhi with Rickettsia prowazekii and Rickettsia conorii revealed only 24 genes unique to R. typhi.

Finally, S. pyogenes strain MGAS10394 is the first Streptococcus M6 genome to be sequenced. The genome is $1899877 \mathrm{bp}$ long, making it the largest Group A
Streptococcus (GAS) genome sequenced to date (Banks et al., 2004). It includes eight prophage-like regions and an $8.3 \mathrm{~kb}$ prophage relic that encodes the SpeA4 variant of $S$. pyogenes exotoxin A. One of the prophage-like elements contains a transposon encoding the mefA gene and a surface-exposed protein (the 'R6 protein', which corresponds to M6_Spy1173) thought to be responsible for the M6 serotype. Interestingly, virtually all the serotype M6 strains examined (104 strains) had this gene, while none of the strains examined with the 11 other M protein serotypes (112 strains) had the R6 gene (Banks et al., 2004). Also, a SpeA4 variant was found, and 19 distinct combinations of prophage element genes encoding
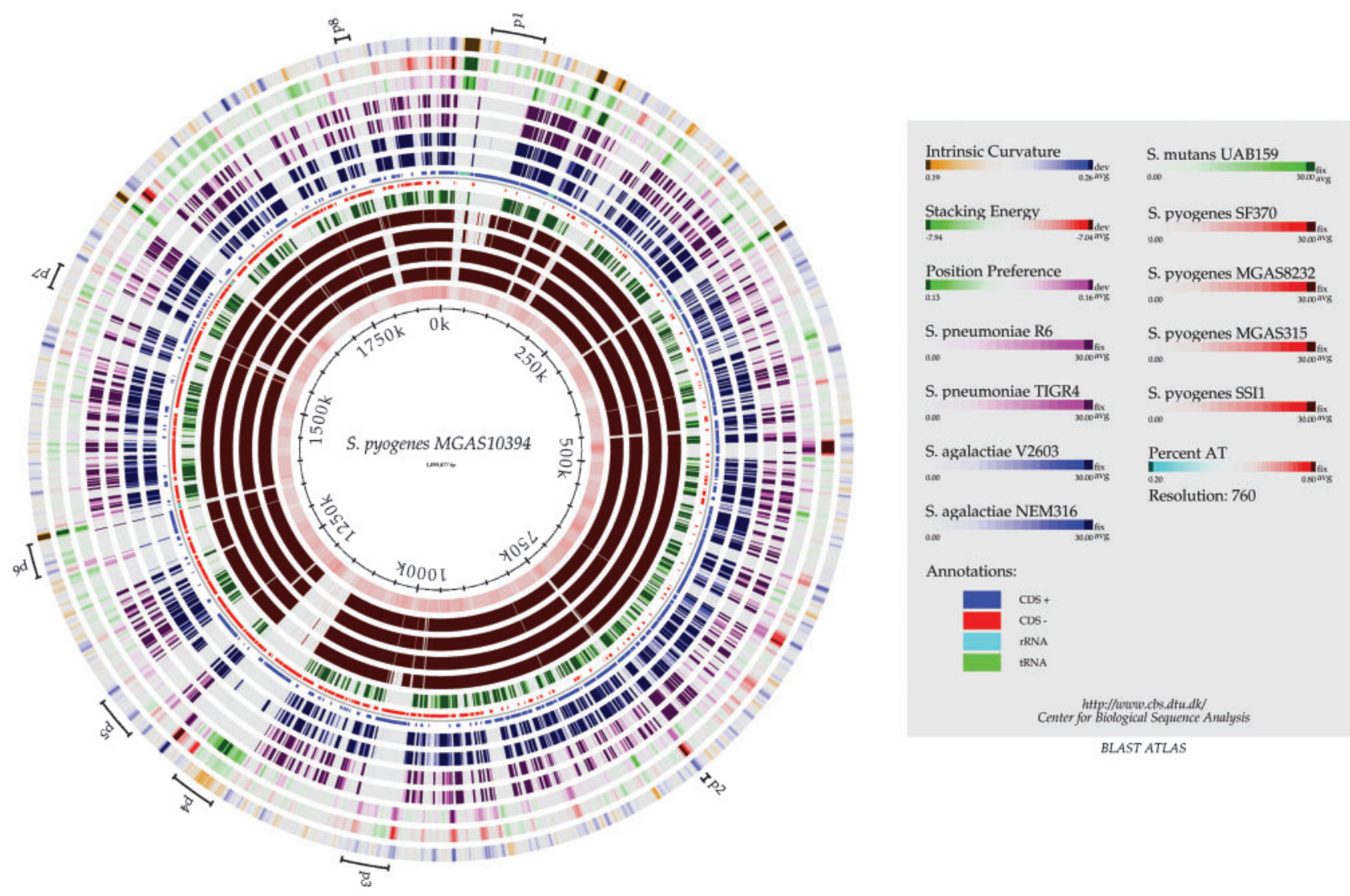

Fig. 1. DNA Atlas plot for the S. pyogenes MGAS10394 chromosome. Each circle represents a different property mapped along the chromosome; for example, the outermost circle plots intrinsic curvature along the sequence, with regions more curved than average in blue, and regions less curved in orange. For the BLAST comparisons, a strong match is darkly coloured; regions with weak or no matches are not coloured. 
proven or putative virulence proteins were present. This result is in agreement with previous work from M1 and M18 strains (Banks et al., 2004).

In addition to the above-mentioned publication concerning the Streptococcus M6 genome sequence, another recent article describes an extensive comparison of 255 different Streptococcus serotype M3 strains, cultured from different patients over a period of 11 years (Beres et al., 2004). A variety of different molecular biology techniques were utilized to explore genetic diversity in these strains (including PFGE, DNA-DNA microarray and prophage genotyping). Variation in gene content (including virulence genes) in different strains can result from acquisition or loss of prophages, and the conclusion of this study is that the assigned M3 genotypes show that phage-induced population changes can be responsible for different severities of infections (Beres et al., 2004).

\section{Method of the month - Chromosome Atlases}

In previous Genome Updates, we have brought forward methods for complete chromosome analysis such as predictions of tRNAs and rRNAs, analysis of AT content, analysis of homology by using the Artemis Comparison Tool (ACT), and promoter profiles. Each method is important in itself, but this month we will use Chromosome Atlases to join different kinds of information (properties) in a single view of the chromosome. This enables a fast visual overview and reveals possible correlations between properties. The genus Streptococcus is well represented (11 strains sequenced from four different species) in the Genome Atlas Database. We have performed a nucleotide BLAST of all ORFs in strain MGAS10394 against nine other Streptococcus chromosomes and mapped all - log E-values along the chromosome, in a colour scale ranging from nearly 0 (no homology) to 30 $\left(E=1 \times 10^{-30}\right)$. (The $S$. pyogenes strain M5 Manfredo was left out since it lacks annotations.) Along with these nine data lanes, AT content, Intrinsic Curvature, Stacking Energy and Position Preference are displayed, as described previously (Pedersen et al., 2000). The Atlas and the lane descriptions can be seen in Fig. 1.
The region marked $\mathrm{P} 4$ is low in intrinsic curvature, position preference and stacking energy, while there is no visible change in AT content compared to the mean value for the chromosome. This could suggest an area of foreign DNA, phage insertion or DNA uptake. The lanes of BLAST results clearly show that $\mathrm{P} 4$ features are not present in any of the genomes except for that of S. pyogenes strain MGAS10394. Phage P1 is only partly present in S. pyogenes strain SSI 1 and S. pyogenes strain MGAS315, while absent in all other chromosomes except that of $S$. pyogenes strain MGAS10394. Phage P2, on the other hand, is present in $S$. pyogenes strains SSI1, MGAS315 and MGAS8232, while it is partly present in strain SF370. Phage P2 is absent in all other species of the genus Streptococcus. The Atlas approach is useful for combining different kinds of information to build a genome comparison. The program used to construct these atlases (GeneWiz) has been implemented in a web-based service, where users can make their own Custom Atlases, at the CBS Genome Atlas Database site http://www.cbs.dtu.dk/services/ GenomeAtlas/myAtlas

\section{Supplemental web pages}

Web pages containing supplemental material related to this article can be accessed from the following url: http:// www.cbs.dtu.dk/services/GenomeAtlas/ suppl/GenUp009/

\section{Acknowledgements}

This work was supported by a grant from the Danish National Research Foundation.

\section{Peter F. Hallin, Tim T. Binnewies and David W. Ussery}

Center for Biological Sequence Analysis, Department of Biotechnology, Building 208, The Technical University of Denmark, DK-2800 Kgs. Lyngby, Denmark

\section{Correspondence: David W. Ussery} (dave@cbs.dtu.dk)

Banks, D. J., Porcella, S. F., Barbian, K. D. \& 7 other authors (2004). Progress toward characterization of the group A Streptococcus metagenome: complete genome sequence of a macrolide-resistant serotype M6 strain. J Infect Dis 190, 727-738.
Beres, S. B., Sylva, G. L., Sturdevant, D. E. \& 11 other authors (2004). Genome-wide molecular dissection of serotype M3 group A Streptococcus strains causing two epidemics of invasive infections. Proc Natl Acad Sci U S A 101, 11833-11838.

Brüggemann, H., Henne, A., Hoster, F., Liesegang, H., Wiezer, A., Strittmatter, A., Hujer, S., Durre, P. \& Gottschalk, G. (2004). The complete genome sequence of Propionibacterium acnes, a commensal of human skin. Science 305, 671-673.

Jaffe, J. D., Stange-Thomann, N., Smith, C. \& 16 other authors (2004). The complete genome and proteome of Mycoplasma mobile. Genome Res 14, 1447-1461.

McLeod, M. P., Qin, X., Karpathy, S. E. \& 19 other authors (2004). Complete genome sequence of Rickettsia typhi and comparison with sequences of other Rickettsiae. J Bacteriol 186, 5842-5855.

Monteiro-Vitorello, C. B., Camargo, L. E., Van Sluys, M. A. \& 41 other authors (2004). The genome sequence of the Gram-positive sugarcane pathogen Leifsonia xyli subsp. xyli. Mol Plant Microbe Interact 17, 827-836.

Pedersen, A. G., Jensen, L. J., Brunak, S., Staerfeldt, H. H. \& Ussery, D. W. (2000). A DNA structural atlas for Escherichia coli. J Mol Biol 16, 907-930.

Rabus, R., Ruepp, A., Frickey, T. \& 15 other authors (2004). The genome of Desulfotalea psychrophila, a sulfate-reducing bacterium from permanently cold Arctic sediments. Environ Microbiol 6, 887-902.

DOI 10.1099/mic.0.27582-0 